\title{
The post-2015 agenda: staying the course in maternal and child survival
}

\author{
Jennifer Harris Requejo, ${ }^{1,2}$ Zulfiqar A Bhutta 2,3,4
}

\begin{abstract}
${ }^{1}$ Partnership for Maternal, Newborn \& Child Health, Geneva, Switzerland ${ }^{2}$ Institute for International Programs, Johns Hopkins Bloomberg School of Public Health, Baltimore, Maryland, USA

${ }^{3}$ Center for Global Child Health, Hospital for Sick Children, Toronto, Ontario, Canada

${ }^{4}$ Center of Excellence in Women and Child Health, Aga Khan University, Karachi, Pakistan
\end{abstract}

\section{Correspondence to} Professor Zulfiqar A Bhutta, Robert Harding Chair in Global Child Health \& Policy, Center for Global Child Health, Hospital for Sick Children, Toronto, Ontario, Canada M5G OA4;

zulfiqar.bhutta@sickkids.ca

Received 18 November 2014 Revised 9 December 2014 Accepted 10 December 2014

\section{(a) CrossMark}

To cite: Requejo JH, Bhutta ZA. Arch Dis Child 2015;100(Suppl 1):s76-s81.

\section{ABSTRACT}

In this article, we draw on available evidence from Countdown to 2015 and other sources to make the case for keeping women and children at the heart of the next development agenda that will replace the Millennium Development Goal (MDG) framework after 2015. We provide a status update on global progress in achieving MDGs 4 and 5, reduce child mortality and improve maternal health, respectively - showing that although considerable mortality reductions have been achieved, many more women's and children's lives can be saved every day through available, cost effective interventions. We describe key underlying determinants of poor maternal and child health outcomes and the need for well-coordinated, comprehensive approaches for addressing them such as introducing a combination of nutrition specific and sensitive interventions to reduce pervasive malnutrition, targeting interventions to the underserved to reduce inequities in access to care, and increasing women's social status through improved access to education and income-earning opportunities. In the wake of population momentum and emergencies such as the recent ebola outbreak and other humanitarian crises, health systems must be strengthened to be able to respond to these pressures. In conclusion, we underscore that the unfinished business of women's and children's health must be prioritized in the days ahead, and that ending preventable maternal and child deaths is not only a moral obligation but is achievable and essential to sustainable development moving forward.

The end of the period of the Millennium Development Goals (MDGs) is fast approaching, with only a little more than 1 year to go. In 2015 , the world will take stock of what has been achieved, and a new global framework to reduce extreme poverty will be introduced building on the lessons learned from the past 15 years. The current MDGs will be replaced by a set of sustainable development goals (SDGs) with a more limited focus on health. The exact nature of global ambition captured in the SDGs and accompanying targets will become apparent by September 2015. It is imperative that the global community maintains the momentum for women's and children's health moving forward to 2015 and beyond, placing women and children firmly where they belong at the centre of the next, more expansive development agenda.

The MDGs, established in 2000, committed the global community to achieve a set of targets by 2015, relative to a baseline of 1990 . At the core of the MDGs are MDG 4, which calls for a reduction of child mortality by two-thirds, and MDG5,

\section{Key messages}

- Maternal and child mortality has dropped nearly $50 \%$ since the 1990 s. However, 17,000 children are still dying every day from preventable causes, stillbirth rates have not measurably changed, and numerous women are dying or suffering from acute or chronic illnesses due to childbirth.

- Malnutrition accounts for nearly half of all child deaths, and places children at risk of both short and long term negative developmental and health outcomes. Poor nutrition prior to and during pregnancy is detrimental to a woman's own health and increases her risk of delivering a low birth weight baby.

- Effective preventive and treatment interventions across the continuum of care for reproductive, maternal, newborn and child health and nutrition are available, and, if implemented at scale, could save thousands of women's and children's lives including the poorest.

- During this final year of the MDGs and the transition period to the SDG era, it is imperative that we stay the course on maternal and child survival so that the momentum gained is not lost. This includes a continued emphasis on supporting the continuum of care through effective policies and programs that create a supportive environment enabling families to be able to access needed care without risking financial catastrophe and for children to develop into healthy, productive adults.

- The RMNCH community must effectively articulate how the well-being of women and children rests at the nexus of all the proposed SDGs, and to push for the adoption in the next global framework of feasible targets for ending preventable maternal, newborn and child deaths. Our future depends on it.

which focuses on improvement of maternal health through a reduction of maternal mortality by threequarters and universal access to reproductive health. ${ }^{1}$ Although substantial progress has been achieved in reducing maternal and child mortality, the world is off track for reaching MDGs 4 and 5 and the rates of reduction for both in many countries is much slower than anticipated with some remarkable exceptions. The number of deaths in children under 5 years of age has declined from around 12.7 million in 1990 to around 6.3 million 
in 2013, an approximate 50\% reduction. $^{2} 3$ And, rates of decline have accelerated in recent years including in many countries in Sub-Saharan Africa and South Asia where child deaths are increasingly concentrated-22 high burden countries in these two regions experienced average annual rates of reduction of $4.4 \%$ or higher (the annual decline required to achieve MDG4 over the full time period 1990-2015) in 2000-2012. ${ }^{4}$ All this good news needs to be tempered by the reality that 17000 children under 5 years of age are still dying every day. ${ }^{2}$ The number of maternal deaths has dropped $45 \%$ from an estimated 523000 deaths in 1990 to 289000 deaths in 2013. While impressive, this decline is far from the MDG target calling for a $75 \%$ reduction. The focus on maternal mortality as a marker for improvement in maternal health masks daunting estimates which indicate that for every one of these 289000 women who died of pregnancy-related causes, at least 20-30 others suffer from acute or chronic morbidity, sometimes with permanent health effects that limit their productivity and physical and mental well-being. ${ }^{6}$

Analysis of the causes of maternal and child deaths shows that substantial numbers of women and children are still dying every day from largely preventable causes, the majority from disadvantaged population groups. Figure 1 shows changes over time in the cause of death structure for children under 5 years of age. The leading causes of postneonatal child deaths remain infectious diseases-pneumonia, diarrhoea and malaria-for which effective preventive and treatment options are available. ${ }^{7}$ Neonatal deaths, in the first 4 weeks of life, are a growing proportion (44\%) of all deaths in children under 5 years of age, and global progress in reducing neonatal mortality is lagging. The toll of stillbirths is approximately 2.6 million every year (uncertainty range, 2.1-3.8 million)-1.2 million (uncertainty range, 0.8-2.0 million) of these deaths occur during labour often due to inadequate care, and there is little evidence of any improvement. ${ }^{8}$

More than half of women who die prematurely from pregnancy and childbirth complications are dying from severe bleeding, pregnancy-induced high blood pressure and infections, conditions that can be effectively managed through high quality delivery and postnatal care. About one in four maternal deaths are caused by pre-existing medical conditions such as malaria, diabetes, obesity and HIV, the health impacts of which can be aggravated by pregnancy. ${ }^{9}$ This cause distribution has been relatively constant for at least the past 10 years and is an indication that much more needs to be done to ensure these conditions are addressed throughout pregnancy and delivery (figure 2).
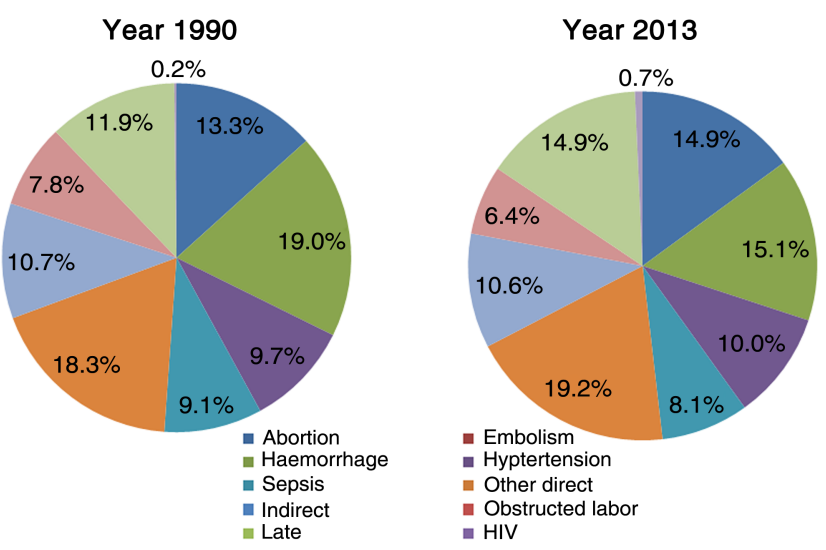

Figure 1 Causes of maternal deaths (Global). ${ }^{3}$

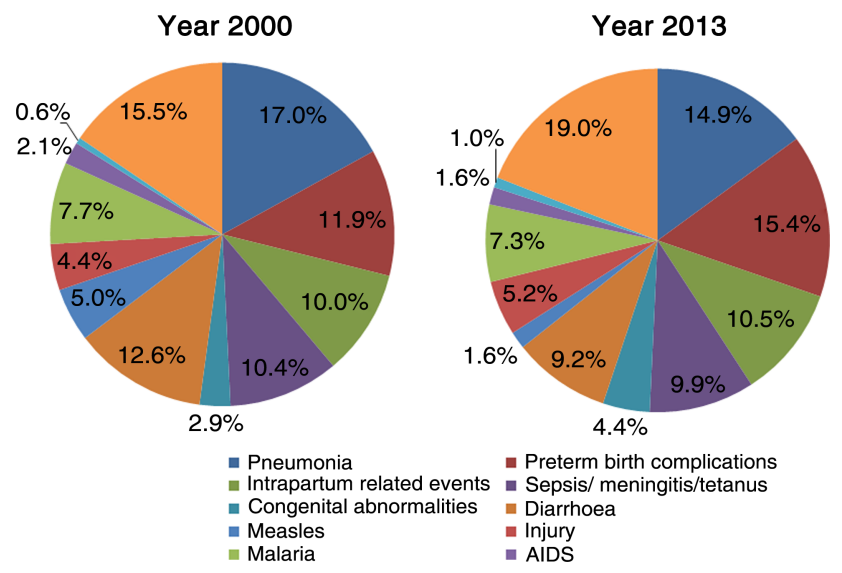

Figure 2 Causes of under 5 deaths (Global). ${ }^{7}$

Malnutrition negatively impacts maternal and child health, with insidious intergenerational effects. Poor nutritional status prior to and during pregnancy is detrimental to a woman's own health and is a risk factor for intrauterine growth restriction and other sub-optimal obstetric outcomes. ${ }^{10}$ Nearly half of all child deaths_-or around 3.1 million deaths per year-are attributable to undernutrition. ${ }^{11}$ Wasting, an indicator of acute food shortage or disease, affects at least 52 million children globally, sharply elevating their risk of death. Stunting is considered the most sensitive indicator of the quality of a child's life and reflects a combination of long-term nutritional deprivation, poor child care or chronic exposure to infection. ${ }^{12}$ Over half of the Countdown to 2015 countries, the 75 countries that account for over 95\% of all maternal and child deaths, with recent data, have stunting levels exceeding 30\%. ${ }^{4}$ Stunting is more common among the poor and other disenfranchised populations, in rural areas and among boys. On average, stunting prevalence is 2.5 times higher among the poorest wealth quintile than among the richest across the Countdown countries. ${ }^{4}$

Recognition of the crucial role that nutrition plays in child health and development, long-term health outcomes, academic achievement and economic productivity led the World Health Assembly to adopt a set of global targets to improve women's and children's nutritional status by 2025. According to the Global Nutrition Report launched in November, 2014, the world is off track for all six targets, a reminder of how much more coordinated effort is needed to achieve these global commitments (table 1). ${ }^{13}$ Greater awareness of the connection between nutrition and sustainable development has also resulted in a surge of long-overdue efforts on improving nutrition during the 'first 1000 days'-the time frame from the beginning of pregnancy to a child's second birthday when nutrition interventions have the potential to vastly improve short-term and long-term health outcomes as well as human capital generation. ${ }^{11} 15$ Emerging evidence about the importance of preconception nutrition for positive reproductive and other health outcomes is generating interest in developing appropriate interventions for the adolescent phase of the life course and preconception care. ${ }^{16}$ Such efforts need to be applauded and supported through 2015 and beyond.

The latest findings from Countdown show the close links between intervention coverage and maternal, newborn and child survival. Countries with higher levels of intervention coverage tend to have lower levels of child mortality and vice versa. ${ }^{17}$ The Countdown analyses also show wide variations in coverage for key interventions across the reproductive, maternal, 


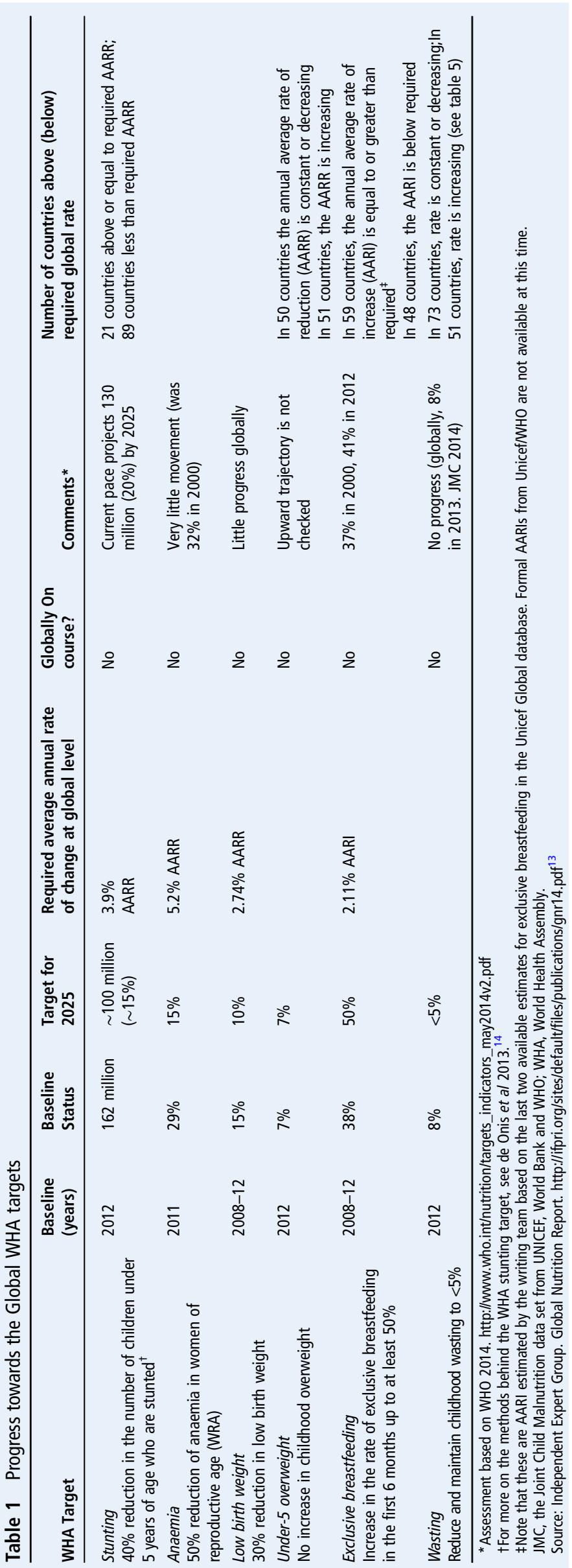

newborn and child health (RMNCH) continuum of care both within and across the 75 countries of priority (figure 3). Gaps in coverage are evident for family planning, care around the time of birth and for case management of childhood diseases such as malaria, pneumonia and diarrhoea-these gaps are alarming given the indisputable evidence of the birth day as the riskiest day for both mother and newborn, that these three infectious diseases account for the bulk of postneonatal deaths, and the protective effect of family planning on maternal and child survival due to improved birth spacing and reduction in higher-order and other high-risk pregnancies. ${ }^{18} 19$ The Countdown equity analyses (figure 4) send a clear message that coverage levels for a set of proven interventions among the wealthiest often far exceeds coverage among the poor. ${ }^{4}{ }^{17}$ Looked at more positively, the attainment of high levels of coverage among the richest in the Countdown countries is proof that these and other low-income and middle-income countries have the capacity to achieve high coverage across the whole population even in the face of resource constraints. It must be underscored that the differentials in terms of coverage for other interventions such as for non-communicable diseases like cancers (eg, cervical and breast) and chronic disorders (eg, sickle cell anaemia) affecting women and children are much greater with many among the poorest at the mercy of ill-equipped and understaffed public sector facilities. ${ }^{20}$ Addressing these inequities must become a priority as the prevalence of non-communicable diseases (NCDs) escalate in low-income and middle-income countries.

\section{PLACING MATERNAL AND CHILD SURVIVAL IN CONTEXT- SOCIAL, ECONOMIC, POLITICAL AND ENVIRONMENTAL FACTORS MATTER}

A major obstacle to progress in intervention coverage faced by many low-income and middle-income countries, particularly those in Sub-Saharan Africa, is continued rapid population growth due to sustained high fertility rates and population momentum. Population increases place considerable strain on often already fragile health systems in these countries to respond to growing demand for services. Although progression through the demographic transition from high to low fertility and mortality rates is complex and is related to improvements in child survival, maternal education and child nutrition, access to family planning, the tools enabling women to control their own fertility, is also a key determinant. A main objective of FP2020, an initiative launched in 2012, is to increase commitment and resources to family planning, particularly in the 69 countries identified as priorities for investment.

Increases in intervention coverage and maternal and child survival cannot happen without vast improvements in health system resilience and critical social and environmental determinants. The recent Ebola outbreak in Guinea, Sierra Leone and Liberia has made irrefutable the importance of ensuring health systems are able to respond to emergencies including epidemics, environmental disasters and humanitarian crises. ${ }^{21}$ Functional health systems must be adequately staffed with sufficiently trained and supervised healthcare workers, consistently equipped with all necessary supplies and equipment and supported by a strong referral chain and health information system for monitoring and planning purposes. The international community has a responsibility to help countries build and sustain such systems and to assist countries experiencing or recovering from conflict situations to reach their populations with needed services. War ravages health systems in both affected and surrounding countries saddled with swells of 


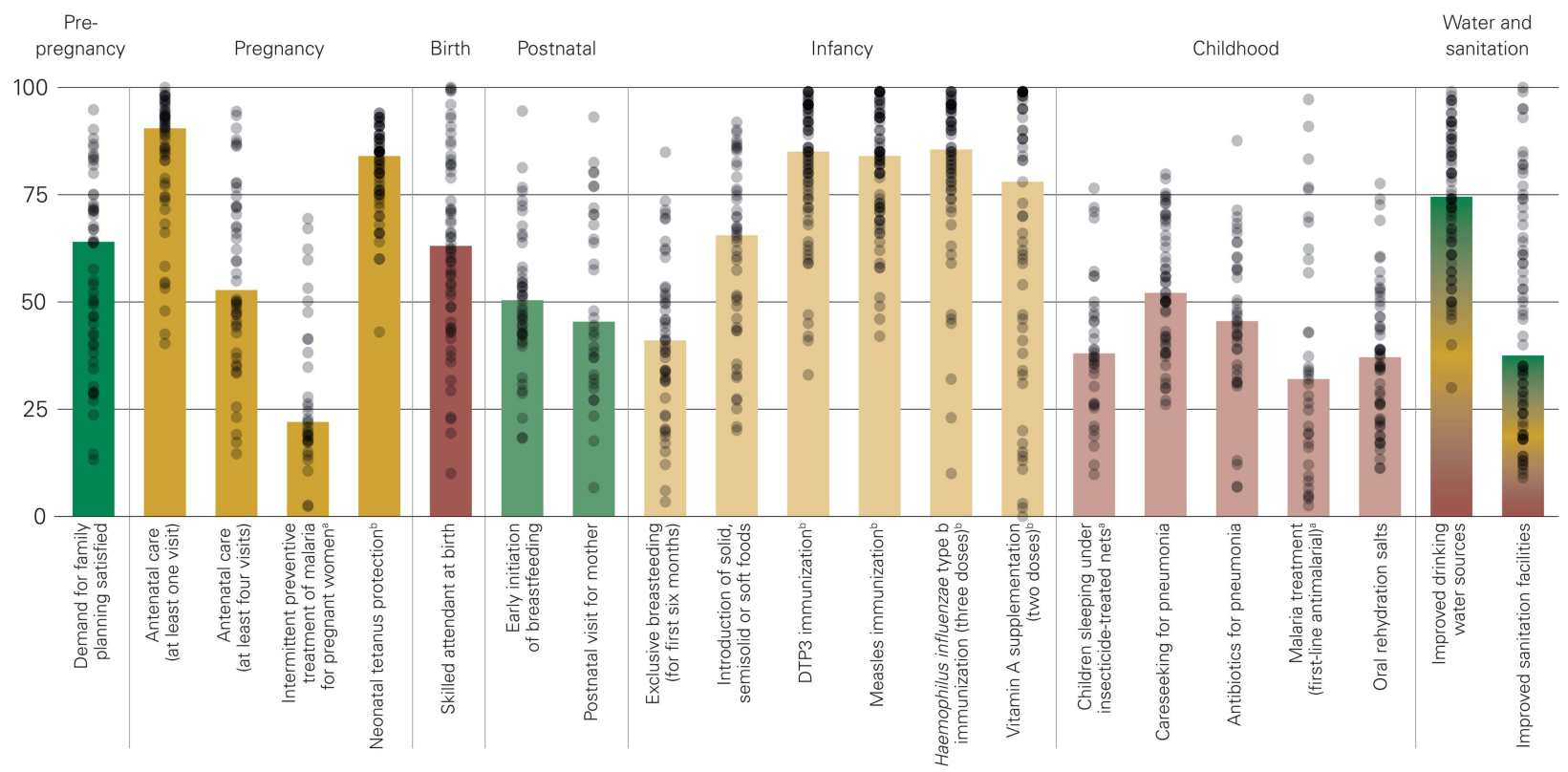

a. Analysis is based on countries with $75 \%$ or more of the population at risk of $p$. falciparum transmission and $50 \%$ or more cases of malaria caused by $p$. falciparum.

b. Data are for 2012 .

Source: Immunization rates, WHO and UNICEF; postnatal visit for mother, Saving Newborn Lives analysis of Demographic and Health Surveys; improved water and sanitation, WHO and UNICEF Joint Monitoring Programme; all other indicators, UNICEF global databases, April 2014, based on Demographic and Health Surveys, Multiple Indicator Cluster Surveys and other national surveys.

Figure 3 The graph showing continuum of care for RMNCH (source: Countdown 2014 report).

refugee populations and turns back the clock on progress in improving maternal and child health. ${ }^{4} 2223$ We may also require strategies for action to support and protect $\mathrm{RMNCH}$ activities in conflict zones and fragile health systems, natural disasters as well as outbreaks.

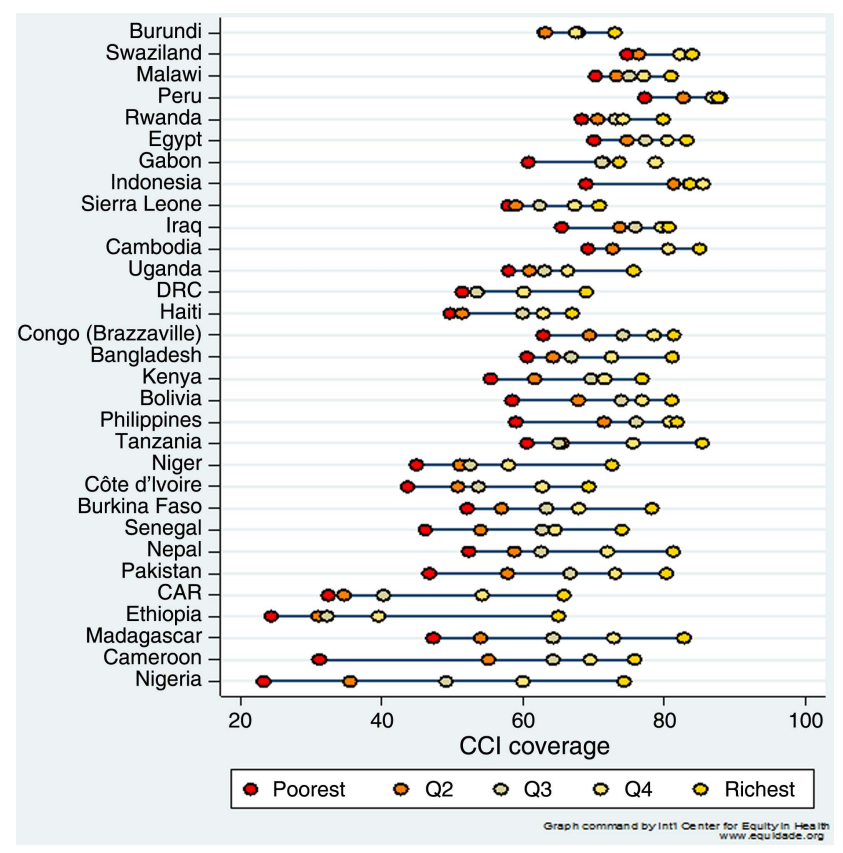

Figure 4 Graph for Composite Coverage Index by wealth quintile (source: Countdown 2014 report).
Numerous reports indicate that many high burden countries are suffering from health workforce crises and decrepit supply chain systems that result in chronic stock-outs of essential drugs, making it impossible for available staff to provide quality care. ${ }^{24}$ Innovations such as cell phones, task shifting practices and expansion of community outreach services are promising approaches to helping countries overcome these challenges, but more investment by governments and their partners is needed to make sure countries are able to provide the basic packages of services needed across the RMNCH continuum of care. ${ }^{22} 2325$ Financial schemes such as cash transfers and the removal of user fees are also essential for increasing the ability of all families to afford care.

According to the WHO, 'Social determinants of health are the conditions in which people are born, grow, live, work and age; these circumstances are shaped by the distribution of money, power and resources at global, national, and local levels'. ${ }^{26}$ The poorest populations tend to be the ones with the least access to care and the highest exposure to infectious diseases and harmful environmental conditions such as overcrowding, air pollution and unimproved water and sanitation facilities. These inequalities are likely to worsen in the wake of population pressures and the havoc produced by climate change. Pervasive gender inequalities and violence against women inhibit women's life opportunities and ability to best care for themselves and their families. Addressing such deep rooted issues requires greater incorporation of human rights and social protection measures into national legal frameworks, specific policies and plans, and long-term investments in girls' education. Gender mainstreaming is another approach that has been used by policy makers when developing budgets and national plans to improve women's social status. 


\section{POST 2015-ACCELERATING THE AGENDA FOR WOMEN AND CHILDREN}

The figures above are a stark reminder that the core business of the MDGs is unfinished and the health of women and children must remain at the heart of the post-2015 Development Agenda. In this final year of the MDGs and during the transition to the SDG era, we must continue working towards achieving high levels of equitable and effective coverage for proven interventions across the $\mathrm{RMNCH}$ continuum of care. This means pushing for integration of services (ie, HIV and malaria services with maternal and child care services) to best leverage resources and to maximise the impact, quality and efficiency of care provided. It also means developing policies and programmes that recognise the tight interlinkages between maternal, newborn and child survival and that are pro-poor, so inequities are steadily tackled as interventions are brought to scale. Implementation of the Every Newborn Action Plan and the Global Action Plan for the Prevention of Pneumonia and Diarrhoea is needed to strengthen country focus on the new born within existing maternal, child and reproductive health plans $^{27}$ and on combatting these two diseases that exact a huge toll of child deaths every year. Support for family planning is essential for empowering women to have more control over their own fertility and for improving maternal and newborn survival-another strategy that embraces the principle of survival convergence and the integrity of the maternal-infant dyad. It will also be important to expand beyond survival to focusing attention on enhancing child and adolescent development and reducing morbidity and the burden of poor mental health.

There are still opportunities to influence the defining of the new goals and targets in the SDG framework. Although there will likely be only one overarching health goal, it is critical that targets for ending preventable maternal, newborn and child deaths and stillbirths are included and clustered together so that the concept of the continuum of care remains intact. A method and a process for disaggregating the data for the health-related targets need to be agreed upon so that the principle of equity is not lost. Feasible targets for mortality reduction are also critical for holding countries to account for progress. Work is also essential in describing the pathways through which other sectors, such as education and agriculture, ultimately impact the health of women and children so that we can achieve each of the stepping stones along these pathways and develop better coordinated multisectoral activities with clearly defined roles and responsibilities.

Echoing the independent Expert Review Group recommendations in its latest report, an independent accountability mechanism for women's and children's health must be put into place post 2015 so that progress can be tracked, gap areas identified and success stories learned from and repeated. ${ }^{28}$ We must all be held to account for doing our part to improve the lives of this and the next generation of women and children. In summary, the SDG framework and associated accountability architecture must:

1. Keep women and children prominently at the centre. Efforts are underway to craft the next Global Strategy for Women's and Children's Health which must articulate how the wellbeing of women and children rests at the nexus of all the SDGs. Recognition of the interconnectedness of women's and children's health to the broader development agenda and the concept of the continuum of care must be retained moving forward so that service integration and effective synergies across sectors happen.

2. Feasible targets for ending preventable maternal, child, newborn mortality and stillbirths need to be prominently featured with associated indicators that promote increased coverage of essential interventions across all population groups. Such targets will help drive coordinated action and accountability for progress.

3. Good nutrition across the life course and particularly during pregnancy and the first 2 years of life must be a priority aspiration. Nutrition specific interventions need to be complemented by nutrition-sensitive approaches that improve access to food, clean water and improved sanitation and social protection measures so that children can survive and thrive.

4. Establish an independent accountability mechanism for women's and children's health that pushes the global community and governments to improve data collection and the use of evidence for decision making. Good data are central to the efficient allocation of resources and for identifying and remedying where progress is lagging. Baseline data must be collected now for the post-2015 era.

5. The international community must be poised to promptly respond to political and environmental crises such as negative effects of climate change to mitigate steep reversals in survival and help countries rebuild. This includes emphasising good governance and dialogue to reduce the risk of armed conflict and civil unrest leading to devastation of the healthcare and educational systems as well as basic infrastructure. Stability is the cornerstone of development, enabling countries to focus resources on this and the next generation.

We are at the cusp of finalising the SDGs post 2015 for which an extensive and inclusive consultative process has been held for well over 2 years. It is quite likely that these goals, targets and indicators will include a broad range of aspirations for global development and inclusion of hitherto neglected areas such as human rights, issues of environment and climate change and non-communicable diseases. We sincerely hope that women and children will remain at the core of the SDGs; it is a global agenda that is far from complete and we have a unique opportunity to achieve reductions in preventable maternal and child mortality within a lifetime.

Acknowledgements We would like to thank members of the Countdown Scientific Review Group, Jennifer Bryce, Cesar Victora, Peter Berman, Joy Lawn, Holly Newby, Andres de Francisco and Monica Fox, for their comments on the paper draft and for giving us permission to use key figures and build on the messages from the latest Countdown to 2015 analyses.

Competing interests None.

Provenance and peer review Commissioned; internally peer reviewed.

\section{REFERENCES}

1 United Nations. Millennium development goals 2014 report. New York: United Nations, 2014. http://www.un.org/millenniumgoals/2014\%20MDG\%20report/MDG \%202014\%20English\%20web.pdf (accessed 15 Nov 2014).

2 UN Inter-agency Group for Child Mortality Estimation. Levels and trends in child mortality: report 2014. New York: United Nations Children's Fund, 2014.

3 Wang $\mathrm{H}$, Liddell CA, Coates MM, et al. Global, regional, and national levels of neonatal, infant and under-five mortality during 1990-2013: a systematic analysis for the Global Burden of Disease Study. Lancet 2014;384:957-79.

4 Countdown to 2015. Fulfilling the health Agenda for women and children: the 2014 report. Geneva: World Health Organization and United Nations Children's Fund, 2014.

5 World Health Organization, United Nations Children's Fund, UNFPA, the World Bank and the United Nations Population Division. Trends in maternal mortality: 1990 to 2013. Geneva: World Health Organization, 2014.

6 Firoz T, Chou D, von Dadelszen P, et al. Measuring maternal health: focus on maternal morbidity. Bull World Health Organ 2013;91:794-6.

7 Liu L, Oza S, Hogan D, et al. Global, regional, and national causes of child mortality in 2000-13, with projections to inform post-2015 priorities: an updated systematic analysis. Lancet 2014. [Publication Online 1 Oct 2014]. doi:10.1016/ S0140-6736(14)61698-6 
8 Lawn JE, Blencowe $\mathrm{H}$, Pattinson $\mathrm{R}$, et al. Stillbirths: Where? When? Why? How to make the data count? Lancet 2011;377:1448-63.

9 Say L, Chou D, Gemmill A, et al. Global causes of maternal death: a WHO systematic analysis. Lancet Global Health 2014;2:e323-33.

10 Bhutta Z, Das J, Rizvi A, et al. Evidence-based interventions for improvement of maternal and child nutrition: what can be done and at what cost?. Lancet 2013;382:452-77.

11 Black R, Victora C, Walker $\mathrm{S}$, et al. Maternal and child undernutrition and overweight in low-income and middle-income countries. Lancet 2013;382:427-51.

12 United Nations Children's Fund. Improving child nutrition: the achievable imperative for global progress. New York: United Nations Children's Fund, 2013

13 Independent Expert Group. Global Nutrition Report. http://www.ifpri.org/sites/ default/files/publications/gnr14.pdf (accessed 15 Nov 2014).

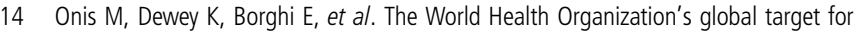
reducing childhood stunting by 2025: rationale and proposed actions. Maternal and Child Nutrition 2013:9:6-26.

15 Victora CG, Adair L, Fall C, et al. Maternal and child undernutrition: Consequences for adult health and human capital. Lancet 2008;371:340-57.

16 Bhutta Z. Beyond Bellagio: addressing the challenge of sustainable child health in developing countries. Arch Dis Child 2004;4:483-7.

17 Requejo J, Bryce J, Barros AJ, et al. Countdown to 2015 and beyond: fulfilling the health agenda for women and children. Lancet 2014. doi:10.1016/S0140-6736(14) 60925-9

18 Saifudin A, Li Q, Liu L, et al. Maternal deaths averted by contraceptive use: an analysis of 172 countries. Lancet 2012;380:111-25.

19 Bhutta ZA, Das JK, Bahl R, et al. Can available interventions end preventable deaths in mothers, newborn babies, and stillbirths, and at what cost? Lancet 2014;384:347-70.
20 Di Cesare M, Young-Ho K, Asaria $\mathrm{P}$, et al. Inequalities in non-communicable diseases and effective response. Lancet 2013;381:585-97.

21 Rid A, Emmanuel EJ. Ethical considerations of experimental interventions in the Ebola outbreak. Lancet 2014:384:1896-9.

22 Bhutta Z, Black R. Global maternal, newborn, and child health — so near and yet so far. N Engl J Med 2013;269:2226-35.

23 Bryce J, Victora C, Black R. The unfinished agenda in child survival. Lancet 2013:382:1049-59.

24 Global Health Workforce Alliance, World Health Organization. Human resources for health: foundation for universal health coverage and the post-2015 development agenda. Report of the Third Global Forum on Human Resources for Health. Geneva: World Health Organization, 2013.

25 Partnership for Maternal, Newborn and Child Health, Aga Khan University, World Health Organization. Essential interventions, commodities and guidelines for reproductive, maternal, newborn and child health: a global review of the key interventions related to reproductive, maternal, newborn and child health. Geneva: Partnership for Maternal, Newborn and Child Health and Aga Khan University, 2011.

26 Marmot M. Closing the health gap in a generation: the work of the Commission on Social Determinants of Health and its recommendations. Global Health Promot 2009;16(Suppl 1):23-7.

27 Starrs $A$. Survival convergence: bringing maternal and newborn health together for 2015 and beyond. Lancet 2014;384:211-3.

28 Independent Expert Review Group. Every Woman, Every Child: A Post-2015 Vision. The third report of the independent Expert Review Group on Information and Accountability for Women's and Children's Health. Geneva: World Health Organization, 2014. 